\title{
ESTIMATING STRESSES IN A PARTIALLY INFLATED HIGH ALTITUDE BALLOON USING A RELAXED ENERGY
}

\author{
$\mathrm{BY}$ \\ WILLIAM G. COLLIER, JR. \\ Department of Mathematics, George Washington University
}

\begin{abstract}
During ascent and at other times during flight, the lifting gas of a high altitude balloon is compressed and only able to partially inflate the balloon. In this condition the surface of the balloon will sag to form folds and wrinkles which are difficult to analyze. Previous numerical work to analyze these types of balloons was based on minimizing extrema of potential energy of balloon shapes that included an explicit representation of excess material as folds. These models used the conventional strain energy for linear isotropic membranes and permitted compressive states to enter the solutions. This paper explores the application of the energy relaxation method to the earlier models to produce solutions free of compressive states. Numerical results computed using the relaxed energy are presented and compared with results computed using the standard strain energy for a membrane.
\end{abstract}

1. Introduction. Early work to model scientific research balloons was done by researchers at the University of Minnesota. Their work focused on fully inflated, inextensible, axisymmetric balloons. More recently, work has been done using finite element methods to treat axisymmetric solutions, see [Sou94], and non-axisymmetric elastic balloons at float altitude, see [Sch91] and [Sch97]. Each of these previous efforts have assumed that the balloon is fully inflated and that the balloon's surface is smooth.

Efforts to study partially inflated balloons mathematically were carried out in [Bag96] and [BR95] using minimization methods to find extrema of the potential energy for the balloon. The models described in these papers handled excess material by introducing an explicit representation of folds forming in the balloon's surface. Both models ignored internal forces in the balloon membrane and included only the weight of components of the balloon and the hydrostatic pressure of the lifting gas. In [BC98], the models in [Bag96] and [BR95] were extended to represent the balloon fabric as an elastic membrane and included elastic cable-like reinforcing elements. This model was revised in [BB98], using

Received July 12, 1999.

2000 Mathematics Subject Classification. Primary 74-xx.

The author was supported by NASA Award NAG5-697.

Current address: Computer Sciences Corporation, 7700 Hubble Dr., Lanham-Seabrook, MD 20706.

E-mail address: wcollier@csc.com 
a different representation of the folded excess material. In the elastic models, [BC98] and [BB98], the balloon's membrane surface was approximated by a collection of triangular planar membranes using the theory of planar elastic membranes. In these models, compressive states developed locally in individual triangles. However, the folds permitted the balloon's surface to sag and relieve, but not eliminate, much of the compressive stress. More precisely, stress resultants averaged across horizontal strips of the balloon's surface were shown to be essentially nonnegative.

In the papers, [Pip86], [Pip94], and [HS94], a theory of membranes has been developed in the context of energy relaxation, as described in [Dac89], that is valid under large deformations. The recent paper, [AS98], expands the application of relaxed energy to coupled problems of elastic membranes with elastic boundaries. The goal of the present work is to apply these relaxed energy theories to the balloon models put forward in [BC98] and [BB98] with the purpose of producing models free of compressive states in partially inflated balloons without the need for calculating stress averages. It has been observed that using energy relaxation methods for treating membranes is closely related to the tension field theory of membranes (see [Pip86] and [Ste91b]). The work by Schur, [Sch97], applied tension field methods to analyzing scientific balloons but did not treat under-inflated cases.

After describing the problem, expressions for the potential energy and relaxed potential energy of a balloon are derived. Then the models presented in [BC98] and [BB98] are summarized along with a scheme for discretizing the energy equations. The effectiveness of this approach is demonstrated by the results of numerical experiments. These experiments produce approximate shapes that are free of compressive states. The numerical results also point out how some of the assumptions made in [BC98] and [BB98] impact the solutions.

\section{Formulation.}

2.1. Problem description. Before trying to develop a mathematical model, a physical description of the scientific balloons under study will help to focus the problem. Scientific balloons are constructed from long tapered panels, called gores, cut from a thin film. Each gore has the same shape and may be over 600 feet long and up to 8 feet wide. The gores are joined together along their edges to form a single enclosure. Often the balloon is outfitted with other structural elements, such as reinforcing tapes along the seams, extra layers of balloon film to reinforce heavily stressed areas, and a top fitting. The balloon assembly becomes a surface in space when inflated with a lifting gas. The shape of the surface is determined by the taper of the gore, the volume of the lifting gas, and the loads acting on the balloon.

The gores are usually tapered to produce a balloon shape with sufficient volume for the buoyant force of the lifting gas to support the balloon and its payload, floating at some determined altitude in the atmosphere. When the lifting gas is compressed into a volume smaller than the intended float volume, the problem is complicated because the entire surface of the balloon film cannot be supported and wrinkles and folds develop. This condition of partial inflation occurs while the balloon is on the ground before launch and during the balloon's ascent after launch. The volume of lifting gas might also be 


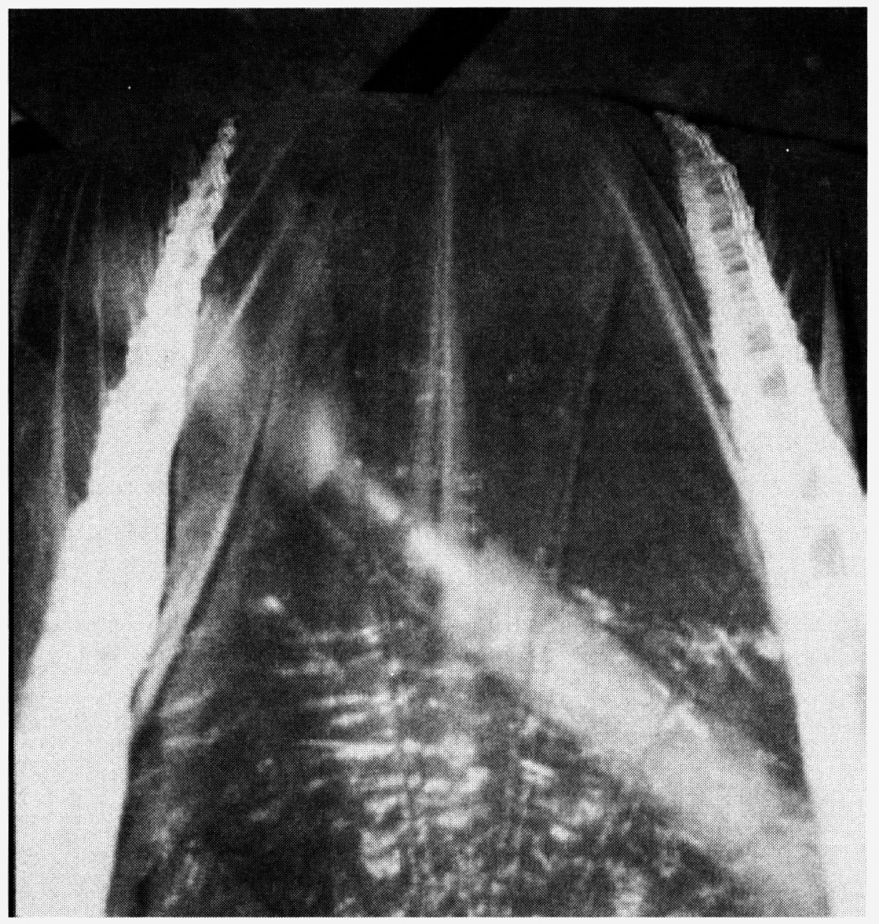

FIG. 1. Fold developing at mid-gore.

reduced while the balloon is near float because of changes in the density of the lifting gas relative to the atmosphere, due to temperature fluctuations during the night and day cycle.

As the volume of lifting gas is reduced, the balloon grows in height and shrinks in girth. Near the apex of the balloon, the gas bubble supports the balloon film with biaxial tension. Below some distance from the apex, the girth of the gas bubble is less than the combined width of all gores at that station. It is unrealistic, in this case, to expect the balloon's film to compress along its circumferential direction in order to meet the girth of the gas bubble, because such a thin film cannot support compression. Instead, the balloon film folds and wrinkles to remove the excess. As a result, the balloon's surface is slack in the circumferential direction and all tension is along the meridian. Even at float altitude, the balloon can be stretched along its vertical axis to create a similar condition. The shape of the under-inflated balloons are often suggestive of dihedral symmetry when viewed directly from above. Observations of real balloons show that for a wide range of volume, most of the excess material gathers by self contact to form a deep fold along a plane of reflectional symmetry at the middle of the gore (see Fig. 1).

The task of modeling the balloon under these conditions is formidable but can be simplified with several assumptions. The material from which a gore is cut is assumed to be linearly elastic and very thin so that the stress normal to the surface is negligible. The reinforcing tapes are represented as linearly elastic cables. The assumptions of linear 
elasticity are reasonable because the stress considered safe for balloon operations is within the linear part of the stress-strain relationship. The balloon is always assumed to be at rest and in static equilibrium. The issue of how this reflects actual stresses in balloons during flight is ignored. The balloon is also assumed to have dihedral symmetry and generated by half of a single gore with half of the reinforcing cable along its outer edge.

The only external forces considered are the hydrostatic pressure of the lifting gas acting on the balloon's surface and the weight of the balloon's membrane, reinforcing cables, and a top fitting. The weight of the payload enters the problem implicitly as the force required to balance the buoyant force and maintain the system in equilibrium with the base of the balloon fixed in space.

2.2. Notation and terminology. In order to describe the problem mathematically, some notation must be developed. The standard inner product on $\mathbf{R}^{3}$ is denoted by $u \cdot v$ where $u, v \in \mathbf{R}^{3}$ and the usual norm is $|u|=(u \cdot u)^{\frac{1}{2}}$. I denotes the $2 \times 2$ identity matrix. The inner product for $2 \times 2$ matrices is $\mathbf{A}: \mathbf{B}=\operatorname{tr}(\mathbf{A B})$, where $\operatorname{tr}$ denotes the trace. For a $2 \times 2$ matrix

$$
\mathbf{A}=\left[\begin{array}{ll}
a_{11} & a_{12} \\
a_{21} & a_{22}
\end{array}\right],
$$

the cofactor matrix, $\operatorname{Cof}(\mathbf{A})$, is defined to be

$$
\operatorname{Cof}(\mathbf{A})=\left[\begin{array}{cc}
a_{22} & -a_{21} \\
-a_{12} & a_{11}
\end{array}\right],
$$

so that

$$
\mathbf{A} \operatorname{Cof}(\mathbf{A})^{T}=\operatorname{det}(\mathbf{A}) \mathbf{I}
$$

(see [Cia88], page 4).

Let $D_{n}$ denote the dihedral group of symmetries of a regular $n$-gon. The action of $D_{n}$ on the plane is denoted by $\chi p$ for $\chi \in D_{n}$ and $p=(X, Z) \in \mathbf{R}^{2}$. This action is extended to isometries of $\mathbf{R}^{3}$ by $\chi r=(\chi p, z), p \in \mathbf{R}^{2}$ and $z \in \mathbf{R}$. If $T$ is a subset of $\mathbf{R}^{3}$ and $\chi \in D_{n}$, then $\chi T$ denotes the set $\{\chi r \mid r \in T\}$.

The surface of the balloon is denoted by $P$. Based on the assumptions stated in Sec. 2.1 , the possible shapes of $P$ are narrowed to those symmetric with respect to $D_{n}$ where $n$ is the number of gores used to construct the balloon. The complete surface is generated by a section, $P_{g} \subset P$, acted upon by $D_{n}$, where $P_{g}$ is a half-gore. It is assumed that the planes

$$
\begin{aligned}
& \Pi_{1}=\{(x, y, z) \mid y=0\}, \\
& \Pi_{2}=\{(x, y, z) \mid x \sin (\pi / n)-y \cos (\pi / n)=0\},
\end{aligned}
$$

are planes of reflectional symmetry for $P$ that bound $P_{g}$. The group $D_{n}$ is generated by two elements, $\left\{\chi_{0}, \chi_{z}\right\}$, where $\chi_{0}$ is the reflection through $\Pi_{1}$ and $\chi_{z}$ is a rotation about the $z$ axis through an angle of $\pi / n$. A whole gore is given by $Q=P_{g} \cup \chi_{0} P_{g}$ and the whole balloon surface is $P=\bigcup_{j=0}^{n-1} \chi_{z}^{j} Q$. Then, $\Pi_{1}$ intersects $P$ along the edge of $P_{g}$ corresponding to the centerline of $Q$, and $\Pi_{2}$ intersects $P$ along the edge of $P_{g}$ with the cable reinforcement.

The reference configuration for the generator of the balloon's surface is a flat unstressed half-gore, $\Omega \subset \mathbf{R}^{2}$. The set, $\Omega$, is situated in the plane so that the mid-gore edge lies 
along the $Z$ axis and the bottom vertex coincides with the origin. The deformation mapping is given by

$$
\phi: \Omega \rightarrow \mathbf{R}^{3}
$$

and $P_{g}$ is the image of $\Omega$ under $\phi$.

Let $\Gamma$ be the boundary of $\Omega$ and $K$ be the boundary of $P_{g}$. The curves $\Gamma$ and $K$ can be broken into two pieces by taking $K_{i}=\Pi_{i} \cap K$ and $\Gamma_{i}=\phi^{-1}\left(K_{i}\right)$, respectively. The curve $\Gamma_{1}$ is a segment of the $Z$ axis and $\Gamma_{2}$ is a simple plane curve. Each curve, $\Gamma_{i}$, has arc length, $L_{i}$. Likewise, each $K_{i}$ is a simple plane curve embedded in $\Pi_{i}$. The curves, $\Gamma_{1}$ and $K_{1}$, correspond to the centerline of the gore while the curves, $\Gamma_{2}$ and $K_{2}$, correspond to the cable running along the edge of the gore. It will be necessary to represent $K_{2}$ parametrically,

$$
\gamma:\left[0, L_{2}\right] \rightarrow \mathbf{R}^{3}
$$

in terms of arc length, $s$, along $\Gamma_{2}$.

2.3. Energy formulation and equations for equilibrium. In this section, a formulation of the balloon problem is presented, using the notation given in Sec. 2.2, as an energy minimization problem.

The thickness of the material in the reference configuration is described by a simple function that is constant over most of the surface and only varies in regions near the top where the membrane is reinforced by multiple layers of material, called caps. Each layer has thickness $\tau$. The first cap covers the region of $\Omega$ consisting of all points

$$
\left\{(X, Z) \mid Z_{1} \leq Z \leq L_{1}\right\}
$$

where $Z_{1}$ satisfies $0<Z_{1}<L_{1}$. The second cap is applied on top of the first cap and covers the points

$$
\left\{(X, Z) \mid Z_{2} \leq Z \leq L_{1}\right\}
$$

with $Z_{2}$ satisfying $Z_{1}<Z_{2}<L_{1}$. It follows that the thickness of a reference half-gore is given by

$$
t(X, Z)=\left\{\begin{array}{c}
\tau: 0 \leq Z \leq Z_{1} \\
2 \tau: Z_{1}<Z \leq Z_{2} \\
3 \tau: Z_{2}<Z \leq L_{1}
\end{array}\right.
$$

for each $(X, Z) \in \Omega$.

The symmetry required for the balloon shape imposes some geometrical constraints on the deformation mapping. The unit normals to $\Pi_{1}$ and $\Pi_{2}$ are denoted by

$$
\begin{aligned}
& \mathbf{N}_{1}=[0,1,0]^{T}, \\
& \mathbf{N}_{2}=[\sin (\pi / n),-\cos (\pi / n), 0]^{T} .
\end{aligned}
$$

A deformation, $\phi: \Omega \rightarrow \mathbf{R}^{3}$, is admissible if it satisfies

$$
\begin{aligned}
0 & \leq \phi \cdot \mathbf{N}_{i}, \quad i=1,2, \\
\phi\left(\Gamma_{i}\right) & \subset \Pi_{i}, \quad i=1,2 .
\end{aligned}
$$

Since the base of the balloon is fixed, a deformation, $\phi$, will also be required to satisfy

$$
\phi(0)=0 .
$$


The class of admissible deformation functions, satisfying the conditions in Equations (2.1), (2.2), and (2.3), is denoted $\mathcal{V}$. Without Eq. (2.1), the balloon's surface would be able to penetrate itself upon reconstructing the whole surface from $P_{g}$. The conditions in Eq. (2.2) are required by symmetry. Later, in Sec. 3, the requirements of Eq. (2.1) will be eased to accommodate the model in [BB98].

In order to describe a partially-inflated balloon with precision, a parameter $\omega_{0}$ is introduced to specify the volume occupied by the lifting gas. The class of admissible functions is then restricted further to allow only surfaces that enclose a given volume $\omega_{0}$. The class of functions in $\mathcal{V}$ that satisfy the volume constraint

$$
2 n \int_{D} d \omega=\omega_{0}
$$

is denoted by $\mathcal{V}_{\omega_{0}}$, where $D$ denotes the three dimensional region bounded by $\Pi_{1}, \Pi_{2}$, and $P_{g}$ and $d \omega$ is used for volume measure on $\mathbf{R}^{3}$. At times it is easier to refer to the volume relative to a reference value, $\omega_{D}$, for the volume of a fully inflated balloon and describe the volume of the lifting gas as a percentage of $\omega_{D}$.

Conditions in Eq. (2.1) introduce inequality constraints into the problem. Let $H_{i}$ be the half-space

$$
H_{i}=\left\{r \in \mathbf{R}^{3} \mid r \cdot \mathbf{N}_{i} \geq 0\right\},
$$

for $i=1,2$. It is clear that the inequalities in Eq. (2.1) require that $\phi(\Omega) \subset H_{i}$, for $i=1,2$. Writing

$$
\phi(V)=\xi_{i}(V)+\left(\phi(V) \cdot \mathbf{N}_{i}\right) \mathbf{N}_{i},
$$

where $\xi_{i}(V) \in \Pi_{i}$, the condition,

$$
\phi(V) \cdot \mathbf{N}_{i}=0
$$

for some $V$ in the interior of $\Omega$ and some for $i=1$ or 2 , shows that $\phi(V) \in \Pi_{i}$. Because of reflectional symmetry, the reflection of $\phi(V)$ through the plane $\Pi_{i}$ must coincide with $\phi(V)$. Thus Eq. (2.4) implies self-contact of the balloon surface in the plane $\Pi_{i}$.

When $i=1$, the contact is near the middle of the gore and one usually sees inward folds such as depicted in Fig. 1. In this case, the contact is on the outer face of the balloon's film. The set $\phi^{-1}\left(\Pi_{1}\right)$ is denoted by $\Omega_{F}$ and is the set of all points in the reference configuration that will be mapped into the fold. For $i=2$, the self-contact occurs in the plane $\Pi_{2}$. What one sees in these cases is not a fold, but material from adjacent gores sucked together and contacting on the inner face of the balloon material. This condition only occurs in lower portions of the balloon. Fig. 2 illustrates the inner self-contact.

The deformation gradient of $\phi$ is the $3 \times 2$ matrix, $\mathbf{F}=\nabla \phi$. There is a decomposition for $\mathbf{F}$ similar to the spectral decomposition for the $3 \times 3$ deformation gradients of three dimension elasticity. The Cauchy strain tensor is the $2 \times 2$ matrix $\mathbf{C}=\mathbf{F}^{T} \mathbf{F}$. Since $\mathbf{C}$ is symmetric and positive-definite, it has a square root $\mathbf{U}$ such that $\mathbf{C}=\mathbf{U}^{2}$. It is then possible to decompose $\mathbf{F}$ as

$$
\mathbf{F}=\mathbf{U Q}
$$

where $\mathbf{Q}=\mathbf{F U}^{-1}$. The $3 \times 2$ matrix $\mathbf{Q}$ has the orthogonality property $\mathbf{Q}^{T} \mathbf{Q}=\mathbf{I}$. 


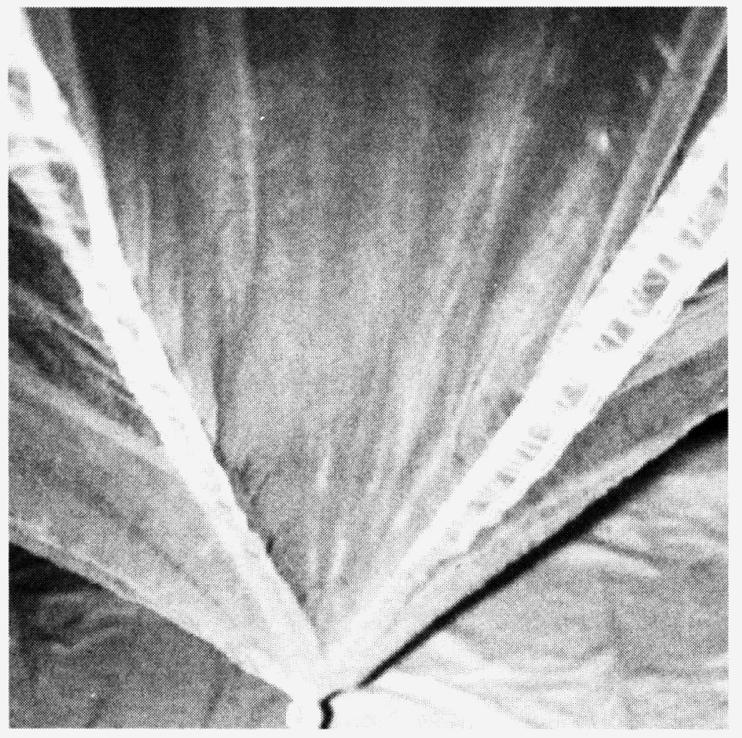

FIG. 2. Contact on face of gore.

The Green's strain tensor is given by

$$
\mathbf{G}=\frac{1}{2}(\mathbf{C}-\mathbf{I}) .
$$

Let $\lambda_{1}^{2}, \lambda_{2}^{2}$ be the eigenvalues of $\mathbf{C}$ so that $\mathbf{U}$ has eigenvalues $\lambda_{1}, \lambda_{2}$. The eigenvalues, $\lambda_{1}, \lambda_{2}$, are called the principal stretches of $\mathbf{F}$ and are positive. It follows that $\mathbf{G}$ has eigenvalues

$$
\epsilon_{i}=\frac{1}{2}\left(\lambda_{i}^{2}-1\right)
$$

Because linear elasticity, isotropy, and zero normal stress for the membrane are assumed, the stress is given by the $2 \times 2$ matrix,

$$
\mathbf{S}=\frac{\Upsilon}{1-\nu^{2}}\left(\mathbf{G}+\nu \operatorname{Cof}(\mathbf{G})^{T}\right)
$$

$\Upsilon$ denotes Young's modulus of elasticity and $\nu$ denotes Poisson's ratio. Writing $\mathbf{S}$ in terms of $\mathbf{G}$ and $\operatorname{Cof}(\mathbf{G})$ will prove useful in keeping expressions succinct. The strain energy of the membrane is given by the expression

$$
W_{f}(\mathbf{G})=\frac{1}{2} \mathbf{S}: \mathbf{G},
$$

which is invariant under the action of $D_{n}$ on $P$.

As an expression in terms of the eigenvalues of $\mathbf{G}$, Eq. (2.6) becomes

$$
W_{f}\left(\epsilon_{1}, \epsilon_{2}\right)=\frac{\Upsilon}{2\left(1-\nu^{2}\right)}\left(\epsilon_{1}^{2}+\epsilon_{2}^{2}+2 \nu \epsilon_{1} \epsilon_{2}\right) .
$$


Substituting the expression in Eq. (2.5) into Eq. (2.7) allows $W_{f}$ to be written as a function of $\lambda_{1}, \lambda_{2}$,

$$
\begin{aligned}
W_{f}\left(\lambda_{1}, \lambda_{2}\right)= & \frac{\Upsilon}{8\left(1-\nu^{2}\right)}\left\{\lambda_{1}^{4}+\lambda_{2}^{4}-(1+2 \nu)\left(\lambda_{1}^{2}+\lambda_{2}^{2}\right)\right. \\
& \left.+2 \nu \lambda_{1}^{2} \lambda_{2}^{2}+(1+2 \nu)\right\} .
\end{aligned}
$$

In the appendix, it is shown that $W_{f}$ is convex as a function of $\epsilon_{1}, \epsilon_{2}$; however, $W_{f}$ is not convex in terms of $\lambda_{1}, \lambda_{2}$. In fact, $W_{f}$ is not convex separately, in either argument, therefore it cannot be rank-one convex and hence not quasiconvex (see [Dac89]).

Straining of the cable is measured by $|\dot{\gamma}|$. The internal energy density function, $W_{c}$, of the cable reinforcement is given in terms of $|\dot{\gamma}|$ by the expression

$$
W_{c}(|\dot{\gamma}|)=\frac{\kappa}{4}\left(|\dot{\gamma}|^{2}-1\right)^{2},
$$

where $\kappa$ is the modulus of elasticity for the cable. $W_{c}$ is also invariant with respect to the action of $D_{n}$ on $P$.

The gravitational forces are easily seen to be conservative and have a potential function, $\Psi(\phi)$, depending only on $\phi$,

$$
\Psi=\int_{P_{y}} \mu_{f} z d A+\int_{K_{2}} \mu_{c} z d s+w_{t} z_{t},
$$

where $\mu_{f}$ is the weight density of the balloon film, $d A$ is surface area measure on $P_{g}, \mu_{c}$ is one-half the weight density of the cable, $d s$ is arc length along $K_{2}, w_{t}$ is the weight of the top fitting, and $z_{t}$ is the $z$ coordinate of the apex of the balloon. The force due to a pointwise pressure distribution, $p$, in a region $D \subset \mathbf{R}^{3}$ is also conservative (see [Fis88], [Ste91a]), and the potential is

$$
\Phi=\int_{D} p d \omega
$$

For hydrostatic pressure,

$$
p(v)=b z
$$

for each $v=(x, y, z) \in \mathbf{R}^{3}$. The real number $b$ is the buoyancy constant for the lifting gas, chosen so that for a given volume, $\omega_{0}$, the magnitude of the buoyant force, $b \omega_{0}$, is equal to the combined weight of the balloon and its payload. It will be shown in Sec. 2.4 that $\Phi$ depends on $\phi$ and $\nabla \phi$. Both the weight functional $\Psi$ and the pressure functional $\Phi$, for hydrostatic pressure, are invariant under the action of $D_{n}$ on $P$.

The total energy for a half-gore is then given by the expression

$$
\begin{aligned}
E_{g}(\phi, \nabla \phi)= & \int_{\Omega} W_{f}(\mathbf{G}) t d \Sigma+\int_{\Gamma_{2}} W_{c}(|\dot{\gamma}|) C d s \\
& +\Phi(\phi, \nabla \phi)+\Psi(\phi) .
\end{aligned}
$$

In this expression, $d \Sigma$ denotes area measure on $\Omega$ and $C$ denotes one-half the cross sectional area of a cable. Because the terms in the expression for $E_{g}$ are invariant with respect to $D_{n}$, it follows that the energy for the whole balloon is

$$
E(\phi, \nabla \phi)=2 n E_{g}(\phi, \nabla \phi) .
$$


Since all of the forces are conservative, equilibrium is achieved at minima of the energy functional, $E$, over the class of functions $\mathcal{V}_{\omega_{0}}$.

Summarizing the result of this section, the equilibrium shape of the balloon sought after in [BC98] and [BB98] is a solution, if such a solution exists, to the following problem:

Problem 1 (Minimization Problem). Find $\phi \in \mathcal{V}_{\omega_{0}}$ such that

$$
E(\phi, \nabla \phi) \leq E(\xi, \nabla \xi)
$$

for all $\xi \in \mathcal{V}_{\omega_{0}}$.

The difference between the model presented in [BC98] and that presented in [BB98] is the exact representation of the fold. This is explained in more detail in Sec. 3.2.

2.4. Relaxed energy. Since $W_{f}$ is not quasiconvex and $W_{c}$ is not convex, the energy function in Eq. (2.11) is not weakly lower semicontinuous, making it unclear whether solutions to Problem 1 exist. It has been shown ([Pip86], [Pip94], [HS94]) that for problems similar to the balloon problem, the associated problem, obtained by replacing the energy densities with their relaxed counterparts, produces solutions that are physically meaningful. The relaxation of $W_{f}$ is its quasiconvexification, the largest quasiconvex function, $W_{f}^{*}$, not exceeding $W_{f}$. Similarly, the relaxation of $W_{c}$ is its convexification, $W_{c}^{*}$, which is the largest convex function not exceeding $W_{c}$. In this section, the idea of energy relaxation is adapted to the balloon problem.

The paper, [AS98], discusses relaxation for problems involving coupling between a thin membrane and an elastic boundary which is applicable to the current problem given the assumptions on the cable reinforcements and their coupling to the balloon film. Following their example, the relaxed energy functional has the form

$$
\begin{aligned}
E_{g}^{*}(\phi, \nabla \phi)= & \int_{\Omega} W_{f}^{*}(\mathbf{G}) t d \Sigma+\int_{\Gamma_{2}} W_{c}^{*}(|\dot{\gamma}|) C d s \\
& +\Phi(\phi, \nabla \phi)+\Psi(\phi) .
\end{aligned}
$$

The functions $\Phi$ and $\Psi$ in Eq. (2.12) are the hydrostatic pressure and weight potentials, respectively, which are the same as Equations (2.9) and (2.10). The weight potential, $\Psi$, is linear in $\phi$ so that this term does not require relaxation. Although the term for the hydrostatic pressure does depend nonlinearly on $\nabla \phi$, it will be shown that this term is already quasiconvex.

In [Pip86] and [Pip94], methods that pertain to $W_{f}^{*}$ are presented for determining the relaxed energy density. These methods lead to a simple characterization of $W_{f}^{*}$ and the further result that $W_{f}^{*}$ is not only quasiconvex but is also a convex and increasing function of $\nabla \phi$. By increasing, it is meant that $W_{f}^{*}(\mathbf{G}+\mathbf{J}) \geq W_{f}^{*}(\mathbf{G})$ for all positive semidefinite square matrices, $\mathbf{J}$. The expression for $W_{f}^{*}$ depends on decomposing the membrane surface of the balloon into three parts, characterized by the state of the membrane. The membrane state is described in terms of the principal values of strain, stress, and their principal directions. The strain and stress tensors are

$$
\begin{aligned}
& \mathbf{G}=\epsilon_{1} n_{1} \otimes n_{1}+\epsilon_{2} n_{2} \otimes n_{2}, \\
& \mathbf{S}=\sigma_{1} n_{1} \otimes n_{1}+\sigma_{2} n_{2} \otimes n_{2},
\end{aligned}
$$


where $\epsilon_{i}$ are the principal strains, $\sigma_{1}=\epsilon_{1}+\nu \epsilon_{2}$ and $\sigma_{2}=\epsilon_{2}+\nu \epsilon_{1}$ are the principal stresses. Since the balloon material is assumed to be isotropic, $\mathbf{G}$ and $\mathbf{S}$ share the same principal directions $n_{1}$ and $n_{2}$. With this notation $P_{g}$ is decomposed into three sets:

$$
\begin{aligned}
& \mathcal{S}=\left\{x \mid \epsilon_{1}<0 \text { and } \epsilon_{2}<0\right\}, \\
& \mathcal{T}=\left\{x \mid \sigma_{1} \geq 0 \text { and } \sigma_{2} \geq 0\right\}, \\
& \mathcal{U}=\{x \mid x \notin \mathcal{T} \text { and } x \notin S\} .
\end{aligned}
$$

The set $\mathcal{U}$ can be broken down further:

$$
\mathcal{U}=\mathcal{U}_{1} \cup \mathcal{U}_{2}
$$

where $\mathcal{U}_{1}=\left\{x \mid \epsilon_{1} \geq 0, \sigma_{2} \leq 0\right\}$ and $\mathcal{U}_{2}=\left\{x \mid \epsilon_{2} \geq 0, \sigma_{1} \leq 0\right\}$. On $\mathcal{S}$, the membrane is slack, $\mathcal{T}$ is where the membrane is taut, and $\mathcal{U}$ is a region where wrinkling is expected. Although $\mathcal{S}$ is admitted as a possibility, in all cases considered in this paper, $\mathcal{S}$ is empty because the entire balloon is under tension in the meridional direction.

The appendix of this paper outlines a derivation of $W_{f}^{*}$, based on [Pip94], completely in terms of the principal values and principal directions of $\mathbf{G}$. Where the membrane is taut (i.e., $x \in \mathcal{T}$ ), $W_{f}^{*}$ is given by Eq. (2.7). When the membrane is slack (i.e., $x \in \mathcal{S}$ ), $W_{f}^{*}$ is 0 . For the case where $x \in \mathcal{U}$, there exists a matrix, $\mathbf{G}^{*}$, such that

$$
\begin{aligned}
W_{f}^{*}(\mathbf{G}) & =W_{f}\left(\mathbf{G}^{*}\right), \\
\mathbf{S}^{*}(\mathbf{G}) & =\mathbf{S}\left(\mathbf{G}^{*}\right) .
\end{aligned}
$$

The matrix $\mathbf{G}^{*}$ can be written as

$$
\mathbf{G}^{*}=\mathbf{G}+\beta^{2} n \otimes n,
$$

where $\beta$ is a real number and $n$ is a unit vector in $\mathbf{R}^{2}$. Pipkin refers to $-\beta^{2} n \otimes n$ as the wrinkling strain and $\mathbf{G}^{*}$ as the elastic strain. The significance of the wrinkling strain is that it modifies the strain to compensate for the slackening of the membrane in the direction of $n$. The elastic strain is thought to represent the strain of some "averaged", unwrinkled surface and leads to uniaxial stress on $\mathcal{U}$,

$$
\mathbf{S}^{*}=\sigma t \otimes t
$$

where $\sigma>0$ and $t$ is a unit vector orthogonal to $n$. The expression for $W_{f}^{*}$ is then

$$
W_{f}^{*}=\left\{\begin{array}{r}
0: x \in \mathcal{S} \\
\frac{\Upsilon}{2} \epsilon_{1}^{2}: x \in \mathcal{U}_{1} \\
\frac{\Upsilon}{2} \epsilon_{2}^{2}: x \in \mathcal{U}_{2} \\
\Upsilon \frac{\Upsilon}{2\left(1-\nu^{2}\right)}\left(\epsilon_{1}^{2}+\epsilon_{2}^{2}+2 \nu \epsilon_{1} \epsilon_{2}\right): x \in \mathcal{T}
\end{array}\right.
$$

The stress function, $\mathbf{S}^{*}$, can be derived from Equations (2.14) and (2.15),

$$
\mathbf{S}^{*}(\mathbf{G})=\lambda\left(\mathbf{G}^{*}+\nu \operatorname{Cof} \mathbf{G}^{* T}\right),
$$

or by differentiation,

$$
\mathbf{S}^{*}=\frac{\partial}{\partial \mathbf{G}^{*}} W_{f}^{*}
$$


In terms of principal values and principal directions, the stress function is

$$
\mathbf{S}^{*}=\left\{\begin{array}{r}
0: x \in \mathcal{S} \\
\Upsilon \epsilon_{1} n_{1} \otimes n_{1}: x \in \mathcal{U}_{1} \\
\Upsilon \epsilon_{2} n_{2} \otimes n_{2}: x \in \mathcal{U}_{2} \\
\sigma_{1} n_{1} \otimes n_{1}+\sigma_{2} n_{2} \otimes n_{2}: x \in \mathcal{T} .
\end{array}\right.
$$

For the cable energy density function, the relaxation of $W_{c}$ is given by the convexification,

$$
W_{c}^{*}=\left\{\begin{aligned}
0:|\dot{\gamma}| & \leq 1 \\
\frac{\kappa}{4}\left(|\dot{\gamma}|^{2}-1\right)^{2}:|\dot{\gamma}| & \geq 1 .
\end{aligned}\right.
$$

The tension in the cable is then given by

$$
T^{*}=\left\{\begin{array}{r}
0:|\dot{\gamma}| \leq 1 \\
\kappa\left(|\dot{\gamma}|^{2}-1\right):|\dot{\gamma}| \geq 1 .
\end{array}\right.
$$

To show that $\Phi$ is quasiconvex, let $\phi_{, 1}=\partial \phi / \partial X$ and $\phi_{, 2}=\partial \phi / \partial Z$. Since $b z$ can be written as the divergence of $\frac{b}{2} z^{2} \hat{k}$, where $\hat{k}$ is the unit vector along the $z$ axis, the divergence theorem gives the hydrostatic potential term as

$$
\begin{aligned}
\Phi(\phi, \nabla \phi) & =\frac{b}{2} \int_{\Omega} z^{2} \hat{k} \cdot n\left|\phi_{, 1} \times \phi_{, 2}\right| d \Sigma \\
& =\frac{b}{2} \int_{\Omega} z^{2} \hat{k} \cdot \phi_{, 1} \times \phi_{, 2} d \Sigma .
\end{aligned}
$$

Eq. (2.22) shows how $P$ depends on $\phi$ and $\nabla \phi$. Define the function

$$
f(u, A)=\frac{b}{2} u_{3}^{2} \hat{k} \cdot \operatorname{adj}_{2} A
$$

for all $u \in \mathbf{R}^{3}$ and all $3 \times 2$ matrices, $A$. In the last expression the notation $\operatorname{adj}_{2} A$ denotes the matrix of determinants of the $2 \times 2$ minors of $A$. Then $f$ is polyaffine in $A$ (see [Dac89], Theorem 1.5), and thus quasiconvex with respect to $A$.

Now Problem 1 can be reformulated in terms of the relaxed energy. Just as in Sec. 2.3 , the energy density functions, $W_{f}^{*}$ and $W_{c}^{*}$, are invariant under the action of $D_{n}$ on $P$, so the total energy is

$$
E^{*}(\phi, \nabla \phi)=2 n E_{g}^{*}(\phi, \nabla \phi)
$$

where $E_{g}^{*}$ is the energy for a half-gore. Then, in view of Eq. (2.12), $E^{*}$ is the functional given by

$$
\begin{gathered}
E^{*}(\phi, \nabla \phi)=2 n\left\{\int_{\Omega} W_{f}^{*}(G) t d \Sigma+\int_{\Gamma_{2}} W_{c}^{*}(|\dot{\gamma}|) C d s\right. \\
+\Phi(\phi, \nabla \phi)+\Psi(\phi)\} .
\end{gathered}
$$

This new energy function, $E^{*}$, is quasiconvex with respect to $\nabla \phi$ and convex in $|\dot{\gamma}|$. The problem now becomes

Problem 2 (Relaxed Minimization Problem). Find $\phi \in \mathcal{V}_{\omega_{0}}$, such that

$$
E^{*}(\phi, \nabla \phi) \leq E^{*}(\xi, \nabla \xi)
$$

for all $\xi \in \mathcal{V}_{\omega_{0}}$. 
3. Discretization. The two mumerical models for partially inflated balloons discussed in [BC98] and [BB98] are discretizations of Minimization Problem 1. This section summarizes the discretization schemes and their application to Problem 2. The key step is approximating the balloon surface with a complex of triangular facets.

Because of the constraints in Eq. (2.1), a portion, $\Omega_{F}$, of the reference surface is expected to satisfy $\phi\left(\Omega_{F}\right) \cdot \mathbf{N}_{1}=0$, while the remaining portion, $\Omega_{O}$, satisfies $\phi\left(\Omega_{O}\right) \cdot \mathbf{N}_{1}>$ 0 . The set, $\Omega_{F}$, is an unknown of the problem and is assumed to be a region that meets $\Omega_{O}$ along a simple plane curve, $\Gamma_{3}$. In the rest of the discussion, the image of $\Gamma_{3}$ in the deformed configuration is denoted by

$$
K_{3}=\phi\left(\Gamma_{3}\right) .
$$

The schemes in [BC98] and [BB98] both determine $\Omega_{F}$ by adjusting nodes on the discrete reference surface that lie along $\Gamma_{3}$, but differ in the way the folds associated with the constraints of Eq. (2.1) are handled in the deformed configuration. The method presented in [BC98] models the condition in Eq. (2.4) explicitly by mapping the fold material, $\Omega_{F}$, into $\Pi_{1}$. The fold is directed inward by assumption and for this reason it is called the interior fold model. In [BB98], $\Omega_{F}$ is mapped into half-space, $\{(x, y, z) \mid y<0\}$, but is no longer subject to the force of pressure from the lifting gas. Here, the essential role of the fold as a mechanism for removing excess material from the balloon's surface is maintained but no attempt is made to represent the fold realistically. Because this model only represents the fold in essence, it is called the virtual fold model.

3.1. The discrete reference configuration. In this section, a discretization of $\Omega$ is presented that includes a subregion $\Omega_{F}$ that will be mapped onto a fold in the deformed surface.

The discretization of the reference configuration, $\Omega$, is the same for both $[\mathrm{BC} 98]$ and [BB98]. Referring to Fig. 3(a), there are $N+1$ vertices, $V_{l, i}=\left(0, Z_{i}\right)$, along the $Z$ axis. These vertices are numbered so that

$$
0=Z_{0}<Z_{1}<Z_{2}<\cdots<Z_{N}=L_{1} .
$$

For $i=1, \ldots, N-1$, there are vertices along the right boundary, $\Gamma_{2}$, of $\Omega$, given by $V_{r, i}=\left(X_{r, i}, Z_{r, i}\right)$ with $Z_{r, i}=Z_{i}$. Let $V_{r, 0}=V_{l, 0}$ and $V_{r, N}=V_{l, N}$. There is still another set of vertices in $\Omega$ represented by $V_{m, i}$, where $i=0, \ldots, N$. Each $V_{m, i}$ lies at the intersection of $\Gamma_{3}$ with the line segment between $V_{l, i}$ and $V_{r, i}$ and is specified by a single parameter $\lambda_{i}$ according to

$$
V_{m, i}=\left(1-\lambda_{i}\right) V_{l, i}+\lambda_{i} V_{r, i} .
$$

The $\lambda_{i}$ lie in the interval $[0,1]$ and specify how much material is committed to the fold. Notice that when $\lambda_{i}$ equals zero, then $V_{m, i}=V_{l, i}$ and there is no fold, while $\lambda_{i}$ equal to one implies that the entire width of the half-gore is folded at that station. The values of the $\lambda_{i}$ are unknown; their determination is part of the solution process.

With the vertices of the triangulation of $\Omega$ now specified, Fig. 3(a) shows how the triangles are layed out on $\Omega$. The set $\Omega_{F}$ is represented by the shaded region of Fig. $3(\mathrm{a})$. The vertices of the triangles in $\Omega_{F}$ belong to the set $\left\{V_{l, i} \mid i=0, \ldots, N\right\} \cup\left\{V_{m, i} \mid i=\right.$ $0, \ldots, N\}$. When $V_{m, i}=V_{l, i}$ for two consecutive values of $i$, there are no triangles in $\Omega_{F}$ for that segment of the gore. This condition is ordinarily found in the upper portion of 
(a)

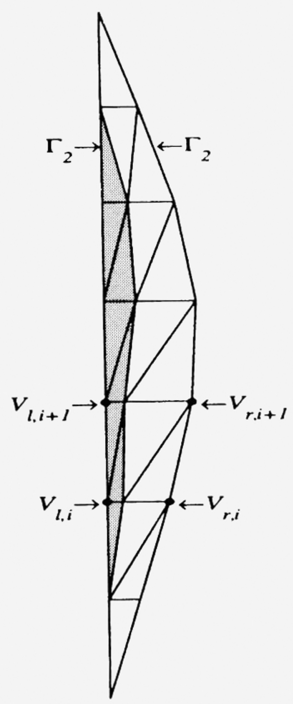

(b)

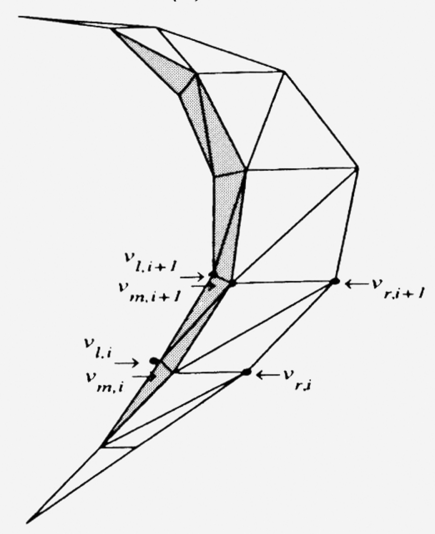

(c)

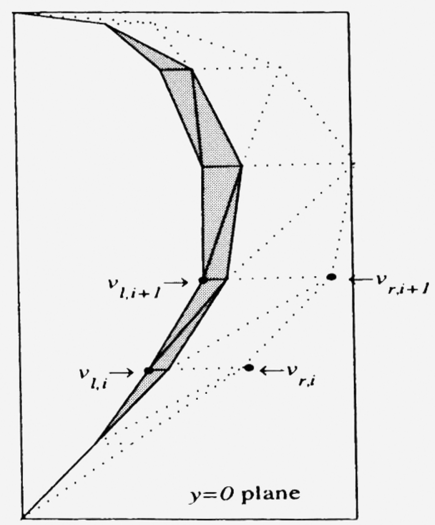

FIG. 3. Discretization of a section of the balloon. (a) Reference configuration $\Omega$, (b) Interior-fold deformation $\phi(\Omega)$, (c) Virtual-fold deformation $\phi(\Omega)$.

the balloon which is occupied by the gas bubble. The unshaded portion of $\Omega$ is $\Omega_{0}$. The vertices of triangles in $\Omega_{0}$ are in the set $\left\{V_{m, i} \mid i=0, \ldots, N\right\} \cup\left\{V_{r, i} \mid i=0, \ldots, N\right\}$.

The mesh appears to be rather coarse across the gore's width. In reality, the gore is much longer than it is wide so that the mesh is actually coarser along its length for typical values of $N$. This quality of the mesh becomes acute at the tapered ends. The mesh in Fig. 3 is presented to illustrate the concepts; details of the actual mesh used in the numerical computations are given in Table 1.

3.2. Discretization of the deformation. In this section, two different schemes are presented for discretizing the deformed balloon surface corresponding to the different models [BC98] and [BB98].

Let $\mathcal{E}$ be the set of all triangles in the mesh. For each $e \in \mathcal{E}$, let $V_{1}^{e}, V_{2}^{e}, V_{3}^{e}$ be the vertices of $e$ in the reference gore and $v_{1}^{e}, v_{2}^{e}, v_{3}^{e}$ the corresponding vertices of the deformed triangle. The discrete deformation mapping is expressed by interpolation on the triangles. If $V \in \Omega$ is in triangle $e$, then

$$
\phi(V)=\sum_{i=1}^{3} v_{i}^{e} M_{i}^{e}(V),
$$

where each $M_{i}^{e}$ is a linear function which satisfies $M_{i}^{e}\left(V_{j}^{e}\right)=1$ if $i=j$ and 0 otherwise.

As already mentioned, the interior fold model maps $\Omega_{F}$ into $\Pi_{1}$. Since the nodes of triangles in $\Omega_{F}$ belong to $\left\{V_{l, i} \mid i=1, \ldots, N\right\} \cup\left\{V_{m, i} \mid i=1, \ldots, N\right\}$, the fold is modeled by constraining $v_{l, i}, v_{m, i} \in \Pi_{1}, i=1, \ldots, N-1$. In order to reduce the number of computations and speed up the process of calculating the energy, the position of $v_{l, i}$ is 
TABle 1. Parameter Values

\begin{tabular}{|l|l|}
\hline Parameter & Value \\
\hline \hline Young's Modulus & $36000 \mathrm{lbs} / \mathrm{in}^{2}$ \\
\hline Poisson's Ratio & 0.82 \\
\hline Balloon Thickness & $0.0008 \mathrm{in}$ \\
\hline $\begin{array}{l}\text { Cable Bulk Modulus } \times \\
\text { Cable Cross Sectional Area }\end{array}$ & $5900 \mathrm{lbs}$ \\
\hline Number of Gores & 159 \\
\hline Number of Caps & 2 \\
\hline$Z_{1}$ & $419 \mathrm{ft}$ \\
\hline$Z_{2}$ & $434 \mathrm{ft}$ \\
\hline$L_{1}$ & $592 \mathrm{ft}$ \\
\hline $\begin{array}{l}\text { maximum width for } \\
\text { half-gore }\end{array}$ & $4.2 \mathrm{ft}$ \\
\hline$N$ & 20 \\
\hline Film Weight Density, $\mu_{f}$ & $2.6667 \times 10^{-5} \mathrm{lbs} / \mathrm{in}^{2}$ \\
\hline Cable Weight Density $\mu_{c}$ & $5.9667 \times 10^{-4} \mathrm{lbs} / \mathrm{in}^{2}$ \\
\hline Top Fitting Weight & $30 \mathrm{lbs}$ \\
\hline Total Payload & $2364 \mathrm{lbs}$ \\
\hline$\omega_{D}$ & $5.0776 \times 10^{10} \mathrm{in}^{3}$ \\
\hline
\end{tabular}

approximated by

$$
\begin{aligned}
\theta_{m, i} & =\frac{v_{m, i+1}+v_{m, i-1}-2 v_{m, i}}{\left\|v_{m, i+1}+v_{m, i-1}-2 v_{m, i}\right\|} \\
v_{l, i} & =v_{m, i}+\alpha_{i} \theta_{l, i},
\end{aligned}
$$

reducing the description of $v_{l, i}$ to the single parameter $\alpha_{i}$. The right hand side of Eq. (3.2) approximates the normal to the plane curve $K_{2}$.

The virtual fold model retains the constraint, $v_{m, i} \in \Pi_{1}$ for $i=1, \ldots, N-1$, but then ignores Inequality (2.1) and allows the nodes $v_{l, i}$ to project through the $x z$ plane along the ray $\overline{v_{r, i} v_{m, i}}$ so that

$$
v_{l, i}=v_{m, i}+\alpha_{i} \frac{v_{m_{i}}-v_{r_{i}}}{\left\|v_{m_{i}}-v_{r_{i}}\right\|} .
$$

This method is motivated by arguing that, because no energy is required to bend the balloon film, the energy functional in Eq. (2.11) should be the same independent of how the material is actually folded. The shaded triangles are now interpreted as excess material that has been folded away in some undetermined way.

In both the interior fold model and the virtual fold model, $\alpha_{i}$ is interpreted as the depth of the fold. Since $\Omega_{F}$ is unknown, the mesh for the reference configuration has to be adjusted, by varying the $\lambda_{i}$ during the solution process, to determine how much material should be mapped into the fold. Ideally, both the $\alpha_{i}$ and the $\lambda_{i}$ would be independent variables. However, another compromise in the numerical procedure was made to reduce 
the number of computations by making each $\lambda_{i}$ a function of $\alpha_{i}$,

$$
\lambda_{i}=\frac{\alpha_{i}}{\alpha_{i}+\left|v_{r, i}-v_{m, i}\right|} .
$$

Some experiments were performed to compare solutions using the approximation in Eq. (3.5) with solutions that treated the variables $\lambda_{i}, i=1, \ldots, N-1$, as independent variables and the differences were not significant.

3.3. Discretization of the energy functional. In this section, expressions are evaluated for the various terms in the relaxed energy functional. In order to avoid a large unwieldy expression, the energy functional is broken into components.

Strain Energy of the Membrane. For each triangle, $e \in \mathcal{E}, V^{e}$ and $v^{e}$ can be interpolated by

$$
\begin{aligned}
V^{e}(\xi) & =V_{0}^{e}+\xi_{1} V_{1}^{e}+\xi_{2} V_{2}^{e}, \\
v^{e}(\xi) & =v_{0}^{e}+\xi_{1} v_{1}^{e}+\xi_{2} v_{2}^{e},
\end{aligned}
$$

where $0 \leq \xi_{1}, \xi_{2} \leq 1, \xi_{1}+\xi_{2}=1$. The Green strain tensor is given by

$$
\mathbf{G}^{e}=\frac{1}{2}\left(\mathbf{F}^{e T} \mathbf{F}^{e}-\mathbf{I}\right) \text {. }
$$

$\mathbf{F}^{e}$ is the deformation gradient, $\nabla \phi$, evaluated on triangle $e$ and is given by

$$
\mathbf{F}^{e}=D v^{e}\left[D V^{e}\right]^{-1}
$$

where $D v=\left[\frac{\partial v^{i}}{\partial \xi_{j}}\right]$ and $D V=\left[\frac{\partial V^{i}}{\partial \xi_{j}}\right]$. The $3 \times 2$ matrix $D v^{e}$ has columns $v_{1}^{e}-v_{0}^{e}$ and $v_{2}^{e}-v_{0}^{e}$ and the $2 \times 2$ matrix $D V^{e}$ has columns $V_{1}^{e}-V_{0}^{e}$ and $V_{2}^{e}-V_{0}^{e}$. The stress is given by

$$
\mathbf{S}^{e}=\lambda\left(\mathbf{G}^{e}+\nu \operatorname{Cof} \mathbf{G}^{e T}\right) .
$$

The strain, $\mathbf{G}^{e}$, and stress, $\mathbf{S}^{e}$, are constant on each triangle and thus each triangle is in exactly one of the states $\mathcal{S}, \mathcal{T}$, or $\mathcal{U}$ everywhere on that triangle. Let $\epsilon_{i}^{e}$ and $\sigma_{i}^{e}, i=1,2$, denote the principal values of $\mathbf{G}^{e}$ and $\mathbf{S}^{e}$, respectively. Then, $W_{f}^{*}$, after being computed as a function of the $\epsilon_{i}^{e}$ and $\sigma_{i}^{e}$, using Eq. (2.17), has a constant value on $e$, denoted by ${ }^{e} W_{f}^{*}$.

The strain energy for the membrane surface of the balloon is

$$
\begin{aligned}
\int_{\Omega} W_{f}^{*}(\nabla \phi) t d \Sigma & =\sum_{e} \int_{\Omega^{e}}{ }^{e} W_{f}^{*} t d \Sigma, \\
& =\sum_{e}{ }^{e} t^{e} W_{f}^{*} \Sigma^{e},
\end{aligned}
$$

where $\Sigma^{e}$ is the area of triangle $e$. Eq. (3.6) is based on the assumption that the mesh was constructed so that $t$ is a constant value ${ }^{e} t$ on each triangle.

Strain Energy of the Edge Reinforcement. Each segment in the discretization of $\Gamma_{2}$ is assigned a label, $a$. Let $V_{0}^{a}$ and $V_{1}^{a}$ denote the endpoints of $a$. The segment $a$ and its deformed image can be interpolated according to

$$
\begin{gathered}
V^{a}(\zeta)=V_{0}^{a}+\zeta\left(V_{1}^{a}-V_{0}^{a}\right), \\
v^{a}(\zeta)=v_{0}^{a}+\zeta\left(v_{1}^{a}-v_{0}^{a}\right),
\end{gathered}
$$


where $\zeta \in[0,1]$. The relative stretch of the cable, $|\dot{\gamma}|$, is approximated by

$$
\left|v_{1}^{a}-v_{0}^{a}\right| /\left|V_{1}^{a}-V_{0}^{a}\right|
$$

Substituting Eq. (3.7) into Eq. (2.20), the quantity $\frac{1}{2}\left(|\dot{\gamma}|^{2}-1\right)$ on segment $a$ is approximated by

$$
\epsilon^{a}=\left\{\begin{array}{r}
0:\left|v_{1}^{a}-v_{0}^{a}\right| /\left|V_{1}^{a}-V_{0}^{a}\right| \leq 1, \\
\frac{1}{2} \frac{\left|v_{1}^{a}-v_{0}^{a}\right|^{2}-\left|V_{1}^{a}-V_{0}^{a}\right|^{2}}{\left|V_{1}^{a}-V_{0}^{a}\right|^{2}}:\left|v_{1}^{a}-v_{0}^{a}\right| /\left|V_{1}^{a}-V_{0}^{a}\right| \geq 1,
\end{array}\right.
$$

and the strain energy of the load tape is

$$
\int_{\Gamma_{2}} W_{c}^{*}(|\dot{\gamma}|) d s=\sum_{a} \kappa C\left(\epsilon^{a}\right)^{2}\left|V_{1}^{a}-V_{0}^{a}\right|
$$

As a matter of practicality, neither $\kappa$ nor $C$ is known individually but the value of the product, $\kappa C$, has been determined experimentally for the edge cable.

Hydrostatic Pressure Potential. To compute the potential energy due to the hydrostatic pressure, let $V_{i}^{e}=\left(X_{i}^{e}, Y_{i}^{e}\right)^{T}$ and $v_{i}^{e}=\left(x_{i}^{e}, y_{i}^{e}, z_{i}^{e}\right)^{T}$, for $i=1,2,3$. Suppose $D$ is the region of $\mathbf{R}^{3}$ bounded by $\Pi_{1}, \Pi_{2}$, and $P_{g}$. Equation (2.22) then becomes

$$
\begin{aligned}
\Phi & =\frac{b}{2} \int_{P_{g}} z^{2} \hat{k} \cdot n d A \\
& =b \sum_{e} \frac{1}{2} \int_{P_{g}^{e}} z^{2} \hat{k} \cdot n^{e} d A \\
& =b \sum_{e}\left(z_{0}^{e 2}+z_{1}^{e 2}+z_{2}^{e 2}+z_{0}^{e} z_{1}^{e}+z_{1}^{e} z_{2}^{e}+z_{0}^{e} z_{2}^{e}\right) A^{e} .
\end{aligned}
$$

In the last expression $A^{e}$ denotes the area of the triangle on the deformed configuration with vertices $v_{1}^{e}, v_{2}^{e}, v_{3}^{e}$, i.e.,

$$
A^{e}=\frac{1}{2}\left|\left(v_{2}^{e}-v_{1}^{e}\right) \times\left(v_{3}^{e}-v_{1}^{e}\right)\right| .
$$

Potential Energy Due to Dead Weight. The terms of the potential for the weight of the various components, using the same notation developed in previous sections, are:

$$
\begin{aligned}
\int_{P_{g}} \mu_{f} z d A & =\sum_{e} \mu_{f} \int_{P_{g}^{e}} z d A \\
& =\sum_{e} \mu_{f} \frac{1}{3}\left(z_{1}^{e}+z_{2}^{e}+z_{3}^{e}\right) A^{e} \\
\int_{\Gamma_{2}} \mu_{c} z d s & =\sum_{a} \mu_{c} \int_{P_{g}^{a}} z d A \\
& =\sum_{a} \mu_{c} \frac{1}{2}\left(z_{1}^{a}+z_{2}^{a}\right)\left|V_{2}^{a}-V_{1}^{a}\right|
\end{aligned}
$$


The combined potential, Eq. (2.9), is then given by

$$
\begin{aligned}
\Psi= & \sum_{e} \mu_{f} \frac{1}{3}\left(z_{1}^{e}+z_{2}^{e}+z_{3}^{e}\right) A^{e} \\
& +\sum_{a} \mu_{c} \frac{1}{2}\left(z_{1}^{a}+z_{2}^{a}\right)\left|V_{2}^{a}-V_{1}^{a}\right| \\
& +w_{t} z_{t} .
\end{aligned}
$$

Volume Functional. The volume of the region, $D$, enclosed by the discrete surface is calculated by summing up the volumes of the tetrahedral subregions. Let $\mathcal{E}_{0}$ denote the set of triangles in $\Omega_{0}$. Then for each $e \in \mathcal{E}_{0}$, a tetrahedron is formed with vertices $\left\{p_{0}, v_{1}^{e}, v_{2}^{e}, v_{3}^{e}\right\}$

$$
D_{p_{0}, v_{1}^{e}, v_{2}^{e}, v_{3}^{e}}=\left\{v \mid v=\beta_{0} p_{0}+\beta_{1} v_{1}^{e}+\beta_{2} v_{2}^{e}+\beta_{3} v_{3}^{e}, \beta_{0}+\beta_{1}+\beta_{2}+\beta_{3}=1\right\} .
$$

The common base point, $p_{0}$, for the tetrahedra is a point on the $z$ axis. Triangles in the fold, $\phi\left(\Omega_{F}\right)$, do not contribute to the volume of $D$. Each $D_{p_{0}, v_{1}, v_{2}, v_{3}}$ has volume

$$
\omega\left(v_{1}, v_{2}, v_{3}\right)=\frac{1}{6}\left(v_{1}-p_{0}\right) \cdot\left\{\left(v_{2}-p_{0}\right) \times\left(v_{3}-p_{0}\right)\right\} .
$$

Summing the individual volumes and multiplying by the number of half-gores, $2 n$, gives the total volume,

$$
\omega(D)=2 n \sum_{e \in \mathcal{E}_{0}} \omega\left(v_{1}^{e}, v_{2}^{e}, v_{3}^{e}\right)
$$

Discretized Minimization Problem. Now that each of the components of the energy functional are determined, they can be summed to obtain an approximation of $E^{*}$ defined in Eq. (2.23). Let

$$
\begin{aligned}
& \mathbf{v}=\left(v_{m, 1}, \ldots, v_{m, N-1}, v_{r, 1}, \ldots, v_{r, N}\right)^{T}, \\
& \mathbf{a}=\left(\alpha_{1}, \ldots, \alpha_{N-1}\right)^{T}
\end{aligned}
$$

and let

$$
\widehat{\mathcal{V}}=\left\{(\mathbf{v}, \mathbf{a}) \mid v_{m, i} \in \Pi_{1}, v_{r, i} \in \Pi_{2}, \alpha_{i} \geq 0, i=1, \ldots, N\right\} .
$$

(Note that $v_{m, 0}, v_{r, 0}$, and $v_{m, N}$ are not included in $\hat{\mathbf{v}}$ because $v_{m, 0}=v_{r, 0}=0$ and $\left.v_{m, N}=v_{r, N}.\right)$ Then $E^{*}=E^{*}(\mathbf{v}, \mathbf{a})$ and $\omega=\omega(\mathbf{v})$.

Problem 3 (Discretized Minimization Problem). Find $(\mathbf{v}, \mathbf{a}) \in \widehat{\mathcal{V}}$ such that

$$
\begin{aligned}
E^{*}(\mathbf{v}, \mathbf{a}) & \leq E^{*}(\mathbf{u}, \mathbf{b}), \\
\omega(\mathbf{v})-\omega_{0} & =0,
\end{aligned}
$$

for all $(\mathbf{u}, \mathbf{b}) \in \widehat{\mathcal{V}}$.

4. Results and discussion. Numerical solutions to Problem 3 were found by encoding $E^{*}$ and $\omega-\omega_{0}$ in the MATLAB programming language and using the MATLAB Optimization Toolbox function CONSTR to determine the minimum of $E^{*}$ as a constrained optimization problem. Solutions were found for a balloon at one hundred percent, ninety percent, and fifty percent of the design volume. The parameters used in these demonstrations are listed in Table 1. 
To obtain solutions at 90 percent and 50 percent of the float volume, it was necessary to begin solving the problem at the float volume and then work down to the desired volume by small decrements. For values near float volume, the numerical procedure was sensitive to changes in the target volume parameter and decrements had to be set to 0.01 percent of the float volume. It appeared that the configuration calculated by the first iteration of CONSTR went too far toward satisfying the volume constraint, producing a severly strained surface for the balloon film. As a result, the strain energy for the first iterate was very large and CONSTR required many iterations before converging to a solution. To work around this problem, families of solutions were calculated by varying the volume parameter slightly and using previous solutions in the family to extrapolate an initial guess for the next solution. As the volume was reduced the volume decrements were increased to values as large as 0.5 percent of float volume.

4.1. Relaxed energy results. As the volume of gas inflating the balloon is compressed, one expects the gas bubble to rise to the top of the balloon, producing biaxial stresses in this region of the balloon film. The lower portion of the balloon should have excess material in the circumferential direction and zero hoop stresses in that vicinity. The results reported in this section confirm these expectations, but the stresses are presented in terms of stress resultants, which are the product of stress and thickness. In Fig. 4, the principal stress resultants for solutions using relaxed energy are presented. Fig. 5 is provided to help correlate the arc length with positions on the balloon. The profiles displayed correspond to the $K_{3}$ curve. The principal directions of the stress resultants are very closely aligned with the circumferential and meridional direction vectors so they have been labeled accordingly. There is uniaxial stress in the lower portion of the balloon with positive stress along the meridional direction. In addition, folds form in the region where there is uniaxial stress. Fig. 6(a) illustrates the depth of the fold for the 50 percent case.

Near the top of the balloon, there is a transition to biaxial stress. The transition for triangles that share an edge with the left boundary of $\Omega_{0}$ occurs lower on the balloon than for triangles sharing an edge with the cable reinforcement. The location of the transition from uniaxial to biaxial stress moves upward as the volume decreases. It is clear that the stress is greater for triangles on the left side of $\Omega_{O}$ in the upper portion of the balloon.

The difference in behavior of the fold depth, in Fig. 6(a), between the interior fold solution and the virtual fold solution can be traced back to the approximations in Equations (3.3) and (3.4). This same effect is present in the 100 percent and 90 percent cases. The interior fold method moves the $v_{l, i}$ nodes inward along the approximate normal to the discretized plane curve, $K_{3}$, at the node $v_{m, i}$. Change of the direction of the normal vector introduces shearing strains. This means the strain energy in the interior fold method is affected by the curvature of $K_{3}$. The curvature is approximated by

$$
\vartheta_{i}^{\prime}=\frac{\vartheta_{i+1}-\vartheta_{i-1}}{\bar{s}_{i+1}-\bar{s}_{i-1}},
$$


(a)

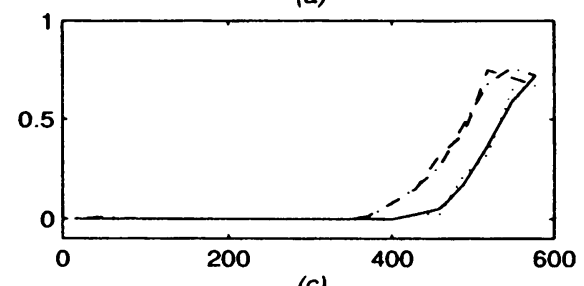

(c)

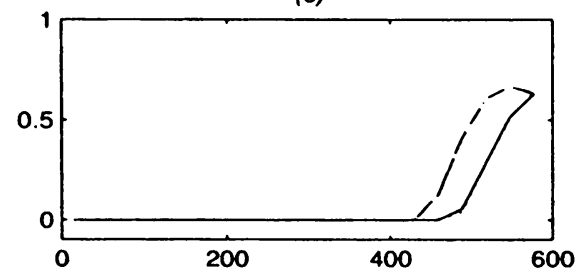

(e)

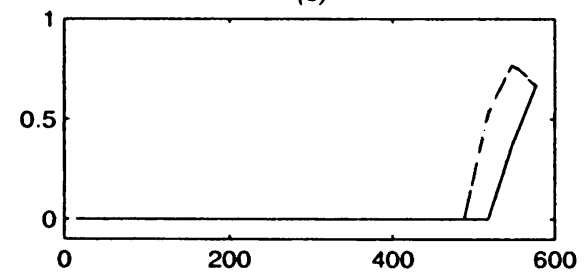

(b)
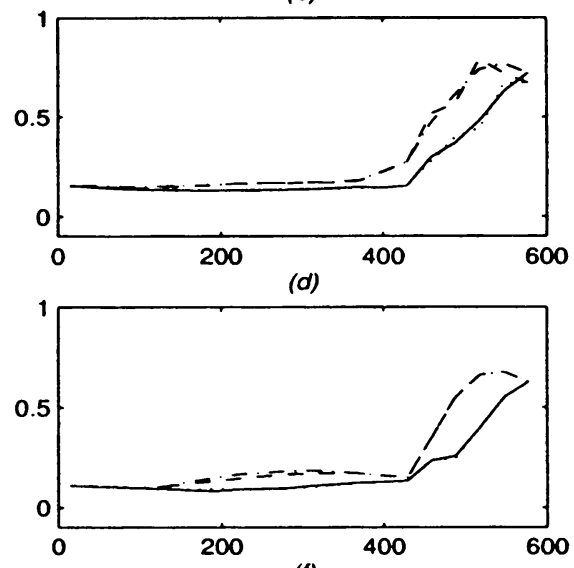

(f)

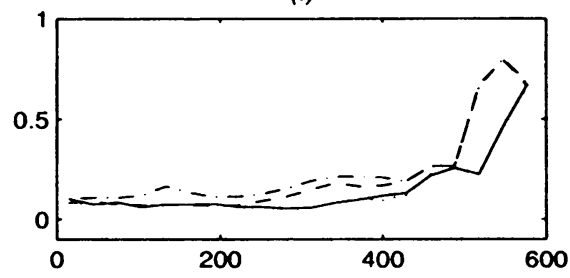

- $\quad$ Stress resultants in left triangles, virtual fold

-.. Stress resultants in right triangles, virtual fold

- - Stress resultants in left triangles, internal fold

Stress resultants in right triangles, internal fold

FIG. 4. Principal stress resultants for the relaxed energy solutions (lbs/in) vs arc length (ft). (a) Circumferential stress at $1.0 \omega_{D}$, (b) Meridional stress at $1.0 \omega_{D}$, (c) Circumferential stress at $0.9 \omega_{D}$, (d) Meridional stress at $0.9 \omega_{D}$, (e) Circumferential stress at $0.5 \omega_{D}$, (f) Meridional stress at $0.5 \omega_{D}$.

where $i=1, \ldots, N-1$. The angles, $\vartheta_{i}$, are defined by

$$
\left[\begin{array}{c}
\cos \vartheta_{i} \\
0 \\
\sin \vartheta_{i}
\end{array}\right]=\theta_{m, i}
$$

where $\theta_{m, i}$ is the approximate normal vector given in Eq. (3.2) and $\bar{s}$ denotes arc length along the mid-gore curve. Fig. 6(a) together with Fig. 6(b) shows a definite relationship between the curvature of the balloon and the fold depth for the interior fold model solutions. Notice that wherever the curvature exceeds $2 \times 10^{-4}$, in absolute value, the fold depth is reduced for the interior fold model. The Virtual Fold Model shows more regular behavior in forming a fold, especially as the folds become deeper.

4.2. Comparative results. In this section the numerical results obtained by using energy relaxation are compared with those obtained using the conventional strain energy. 


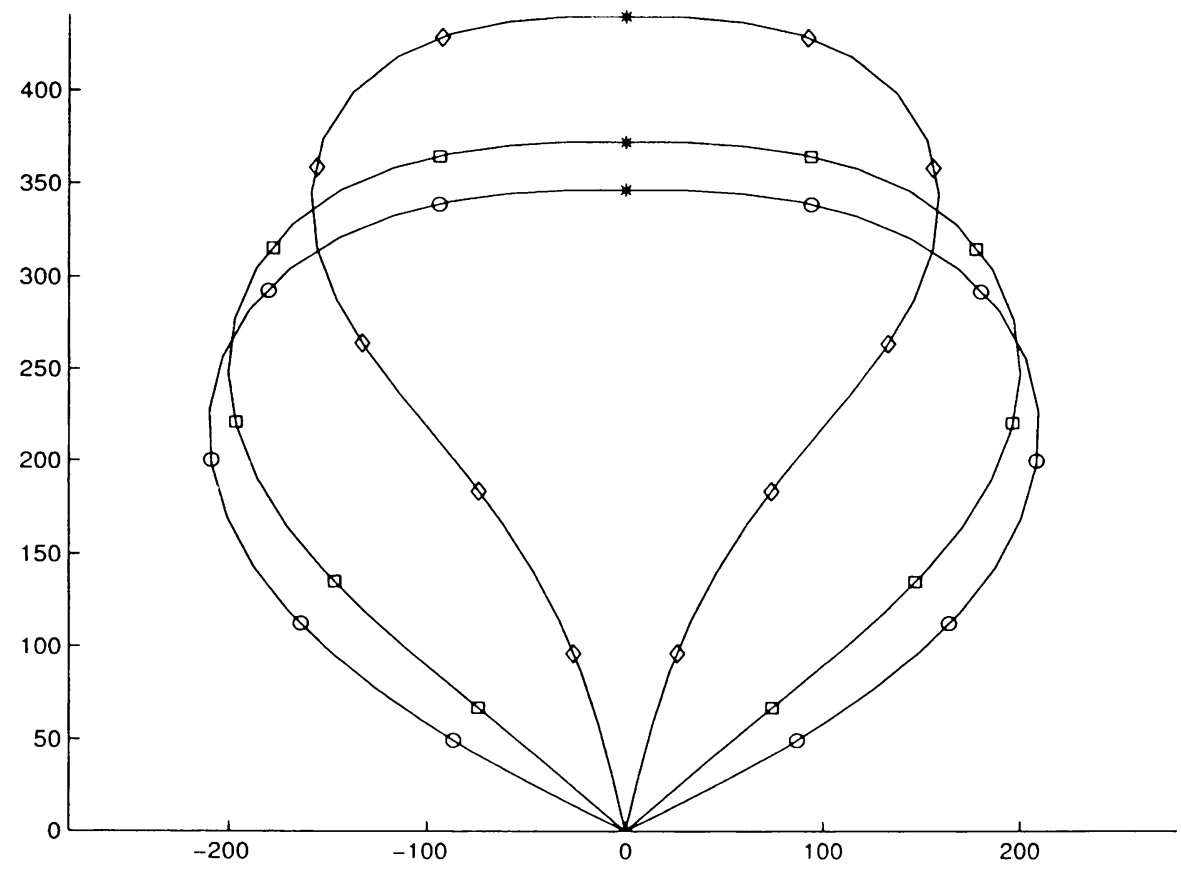

FIG. 5. Balloon profiles (ft). Arc length is marked at $100 \mathrm{ft}$. increments, starting from the base of the balloon. Peak is indicated with a star. Circles mark the $1.0 \omega_{D}$ profile, squares mark the $0.9 \omega_{D}$ profile, and diamonds mark the $0.5 \omega_{D}$ profile.
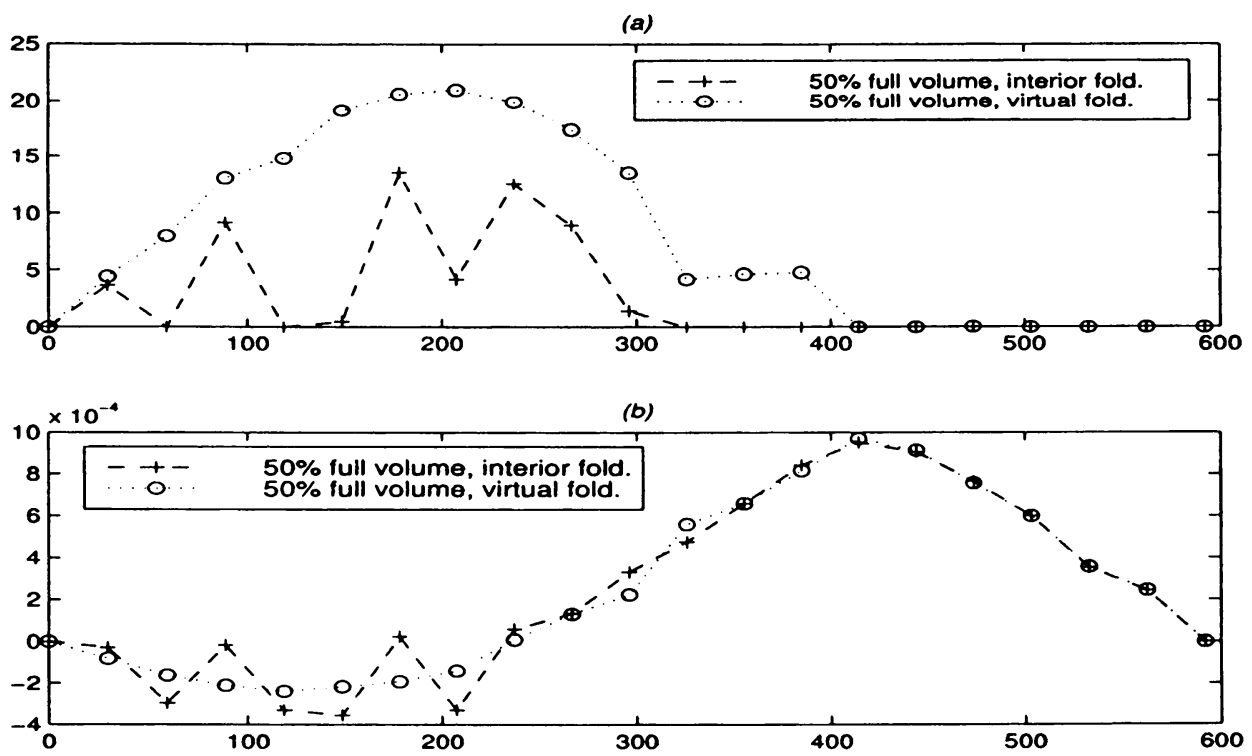

FIG. 6. Fold Depth (in) and Curvature of the outer balloon surface vs arc length (ft) for a volume of $0.5 \omega_{D}$. (a) Fold Depth, (b) Curvature of Fold. 
Since solutions using the standard strain energy exhibit negative values for the circumferential stress resultants, the averages of the principal values of the stress resultants were reported in [BC98] and [BB98]. These averages were essentially nonnegative for the virtual fold model. The averages were calculated according to the following scheme. For each $i=1, \ldots, N-1$, there are adjacent triangles in $\Omega_{O}, \Delta_{i}^{l}$, and $\Delta_{i}^{r} . \Delta_{i}^{l}$ has vertices $\left\{V_{m, i+1}, V_{r, i+1}, V_{m, i}\right\}$ and has an edge along the mid-gore boundary, $\Gamma_{3} . \Delta_{i}^{r}$ has vertices $\left\{V_{r, i}, V_{r, i+1}, V_{m, i}\right\}$ and shares an edge with the cable boundary, $\Gamma_{2}$. Let $R_{i}^{l}$ and $R_{i}^{r}$ denote the stress resultants on $\Delta_{i}^{l}$ and $\Delta_{i}^{r}$, respectively. Then the averaged stress resultants are

$$
R_{i}=\frac{1}{2}\left(R_{i}^{l}+R_{i}^{r}\right)
$$

for $i=1, \ldots, N-1$. Define $R_{0}$ and $R_{N}$ to be the stress resultants of the triangles in $\Omega_{O}$ containing $V_{0}$ and $V_{N}$ so that $R_{i}$ is defined for all $i=0, \ldots, N$. The principal values of $R_{i}$ are denoted by $\delta_{i}^{m}$ and $\delta_{i}^{c}$. The principal directions associated with the $\delta_{i}^{m}$ are found to be aligned close to the meridional direction and the principal directions associated with the $\delta_{i}^{c}$ are close to the circumferential direction.

Figures 7 and 8 show the principal values of $R_{i}$ for the relaxed energy solutions superposed on the values for the conventional strain energy solutions. Fig. 7 shows that the stress resultants for the conventional energy solutions become more irregular and differ from the relaxed strain energy solutions as the volume is decreased in the interior fold model. There is much better agreement between the relaxed energy solutions and the conventional strain energy solutions for the virtual fold model shown in Fig. 8 . There is some minor disagreement in the meridional stress resultants in the interval $[200,450]$, but the circumferential stress resultants are in very good agreement.

5. Concluding remarks. Energy relaxation was applied to two models of partiallyinflated balloons that were formulated from the conventional membrane strain energy. Through numerical experiments, the relaxed models were shown to exhibit some of the most important qualitative features observed in real balloons: regions of excess material in the balloon's surface, absence of compressions, uniaxial stress in regions of excess material, and biaxial stress at the top of the balloon. The models using conventional strain energy handled excess material by "folding" it away. The models with relaxed energy retained these features and developed significant folds even though the solutions were not required to fold. Models that used energy relaxation showed themselves to be much less sensitive to the details of how excess material was modeled in the balloons than the previous models that relied on conventional expressions for strain energy in a membrane.

Appendix: Equations for wrinkle strain. In this appendix, the expressions given in Sec. 2.4 for the relaxed strain and stress associated with linear isotropic material are verified. The notation presented in Sections 2.3 and 2.4 for the stress and strain tensors and their components is assumed. As before, $\sigma_{i}, \epsilon_{i},(i=1,2)$ are the principal stresses and strains, respectively, and $n_{i},(i=1,2)$ are the corresponding normalized principal directions. 

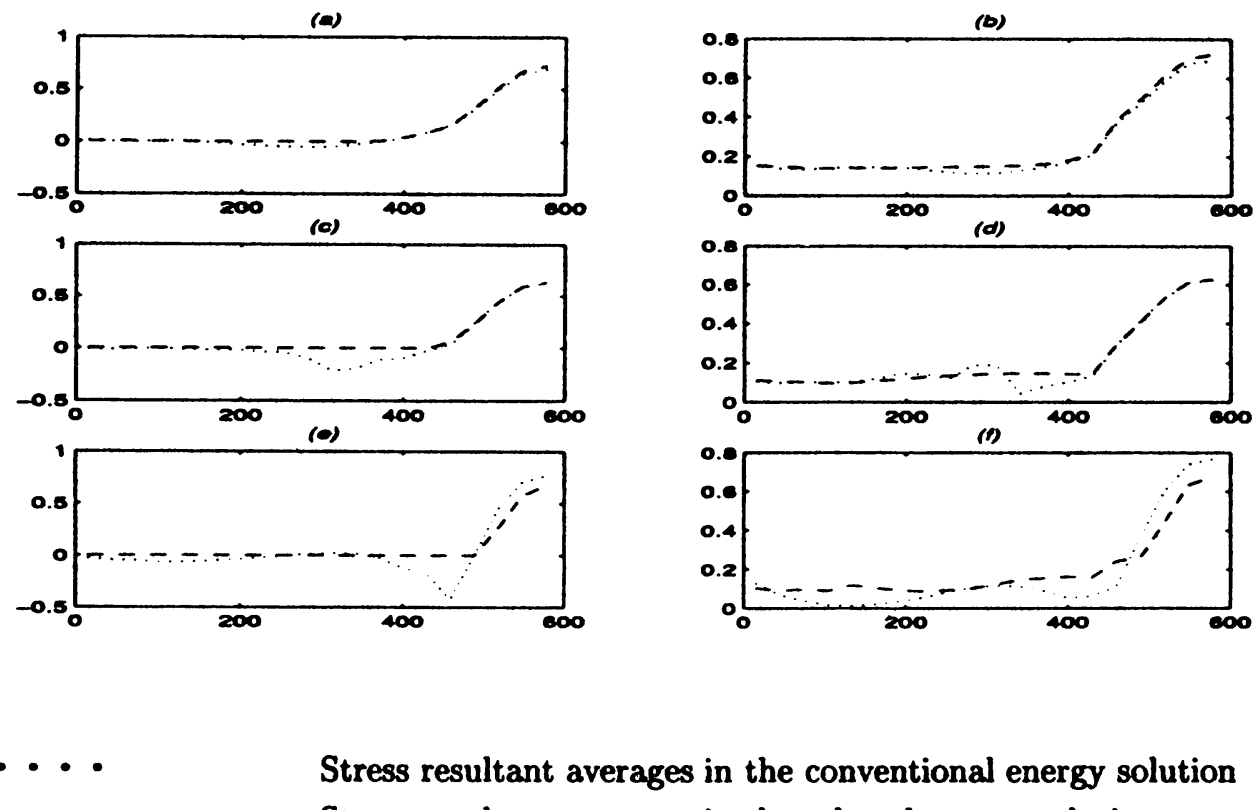

Stress resultant averages in the conventional energy solution Stress resultant averages in the relaxed energy solution

FIG. 7. Superposition of stress resultants (lbs/in) vs arc length (ft). Calculated for internal fold method. (a) Circumferential stress at $1.0 \omega_{D}$, (b) Meridional stress at $1.0 \omega_{D}$, (c) Circumferential stress at $0.9 \omega_{D}$, (d) Meridional stress at $0.9 \omega_{D}$, (e) Circumferential stress at $0.5 \omega_{D}$, (f) Meridional stress at $0.5 \omega_{D}$.

From the expression for $W_{f}$ given by Eq. (2.7), it follows that the convexity of $W_{f}$ as a function of $\epsilon_{1}$ and $\epsilon_{2}$ is equivalent to convexity of the function $\Psi(x, y)=x^{2}+y^{2}+2 \nu x y$. After some manipulation, $\Psi(x, y)$ can be shown to satisfy

$$
\begin{aligned}
& \Psi\left(\alpha x_{1}+\beta x_{2}, \alpha y_{1}+\beta y_{2}\right)=\alpha \Psi\left(x_{1}, y_{1}\right)+\beta \Psi\left(x_{2}, y_{2}\right) \\
& \alpha \beta\left\{\left(x_{2}-x_{1}\right)^{2}+\left(y_{2}-y_{1}\right)^{2}+2 \nu\left(x_{2}-x_{1}\right)\left(y_{2}-y_{1}\right)\right\},
\end{aligned}
$$

where $\alpha+\beta=1, \alpha, \beta \geq 0$, and $0 \leq \nu \leq 1$. Since $2 \nu\left(x_{2}-x_{1}\right)\left(y_{2}-y_{1}\right) \leq 2\left|x_{2}-x_{1}\right|\left|y_{2}-y_{1}\right| \leq$ $\left(x_{2}-x_{1}\right)^{2}+\left(y_{2}-y_{1}\right)^{2}$, the bracketed expression on the right hand side of Eq. (A.1) is positive. It follows that $\Psi$ is convex.

The following two expressions, conclusions of Eq. (2.16), are useful for determining $W_{f}^{*}$ :

$$
\begin{gathered}
n \cdot \mathbf{S}^{*} n=0, \\
n \cdot \mathbf{S}^{*} t=0 .
\end{gathered}
$$

Equation (A.2) is required to assure uniaxial stress in the $t$ direction, while (A.3) is true because $n$ and $t$ are principal directions for $\mathbf{S}^{*}$.

The key step in determining $\beta^{2}$ and $n$ is using Eq. (2.18) to arrive at an expression for $\mathbf{S}^{*}$. Let

$$
\mathbf{K}=\lambda\left(n \otimes n+2 \nu \operatorname{Cof}(n \otimes n)^{T}\right)
$$



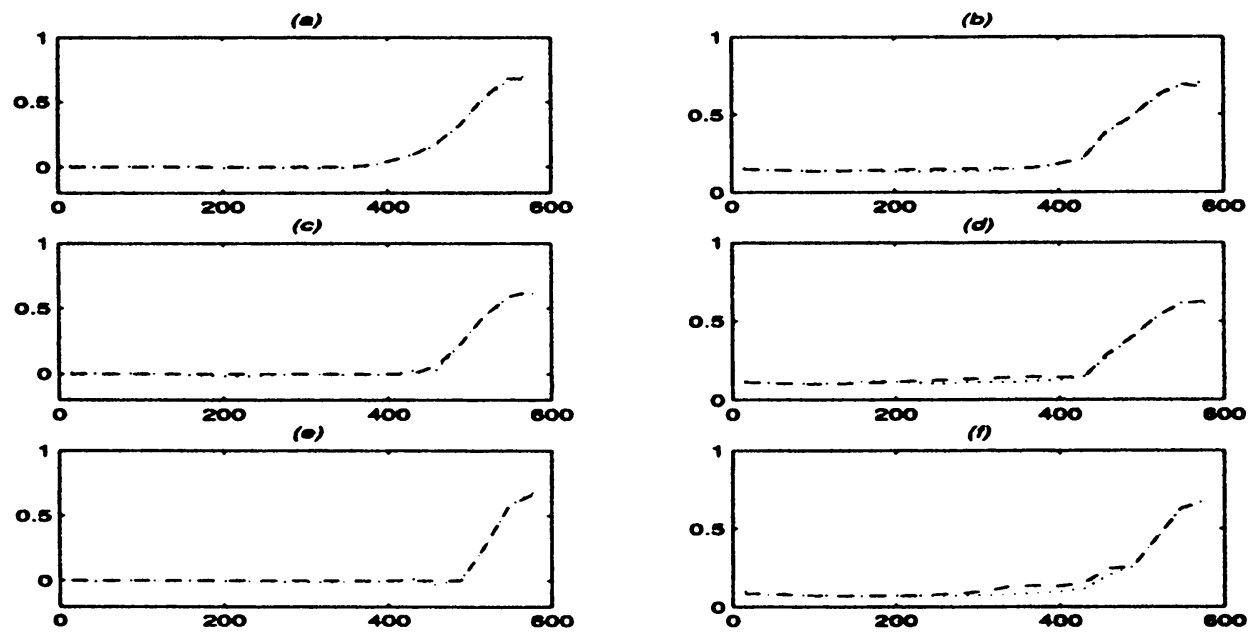

Stress resultant averages in the conventional energy solution Stress resultant averages in the relaxed energy solution

FIG. 8. Superposition of stress resultants (lbs/in) vs arc length (ft). Calculated for virtual fold method. (a) Circumferential Stress at $1.0 \omega_{D}$, (b) Meridional Stress at $1.0 \omega_{D}$, (c) Circumferential Stress at $0.9 \omega_{D}$, (d) Meridional Stress at $0.9 \omega_{D}$, (e) Circumferential Stress at $.05 \omega_{D}$, (f) Meridional Stress at $0.5 \omega_{D}$.

then, by Equations (2.15) and (2.18),

$$
\mathbf{S}^{*}=\mathbf{S}+\beta^{2} \mathbf{K}
$$

After some algebra, the following identities are obtained:

$$
\begin{aligned}
\operatorname{Cof}(n \otimes n)^{T} & =t \otimes t, \\
\mathbf{K} & =\lambda n \otimes n+2 \nu \lambda t \otimes t, \\
\mathbf{K} n & =\lambda n, \\
\mathbf{K} t & =2 \nu \lambda t .
\end{aligned}
$$

Equations (A.5) and (A.6) can be combined with Equations (A.2), (A.3), and (A.4) to show that

$$
\beta^{2}=-\frac{1}{\lambda}(n \cdot \mathbf{S} n)
$$

If $x \in\left\{\epsilon_{2} \geq 0, \sigma_{1} \leq 0\right\}$, then one must take $t=n_{2}$ and $n=n_{1}$. It follows that $\beta^{2}=$ $-\left(\epsilon_{1}+\nu \epsilon_{2}\right)$. If $x \in\left\{\epsilon_{1} \geq 0, \sigma_{2} \leq 0\right\}$, then one has $t=n_{1}, n=n_{2}$, and $\beta^{2}=-\left(\epsilon_{2}+\nu \epsilon_{1}\right)$. 
The conclusion is

$$
\mathbf{G}^{*}=\left\{\begin{array}{c}
0: x \in \mathcal{S} \\
\epsilon_{2}\left(n_{2} \otimes n_{2}-\nu n_{1} \otimes n_{1}\right): x \in\left\{\epsilon_{2} \geq 0, \sigma_{1} \leq 0\right\} \\
\epsilon_{1}\left(n_{1} \otimes n_{1}-\nu n_{2} \otimes n_{2}\right): x \in\left\{\epsilon_{1} \geq 0, \sigma_{2} \leq 0\right\} \\
\epsilon_{1} n_{1} \otimes n_{1}+\epsilon_{2} n_{2} \otimes n_{2}: x \in \mathcal{T} .
\end{array}\right.
$$

From Eq. (A.8) it follows that the principal strains for $\mathbf{G}^{*}$ are

$$
\begin{aligned}
& \{0,0\} \text { if } x \in \mathcal{S} ; \\
& \left\{\epsilon_{2},-\nu \epsilon_{2}\right\} \text { if } x \in\left\{\epsilon_{2} \geq 0, \sigma_{1} \leq 0\right\} ; \\
& \left\{\epsilon_{1},-\nu \epsilon_{1}\right\} \text { if } x \in\left\{\epsilon_{1} \geq 0, \sigma_{2} \leq 0\right\} ; \text { and } \\
& \left\{\epsilon_{1}, \epsilon_{2}\right\} \text { if } x \in \mathcal{T} .
\end{aligned}
$$

Eq. (2.17) comes by evaluating Eq. (2.13) and making use of Eq. (2.7) or by evaluating Eq. (2.14). Eq. (2.19) is obtained by differentiating Eq. (2.17).

Acknowledgement. The work presented here is part of the author's Ph.D. thesis at George Washington University.

\section{REFERENCES}

[AS98] A. A. Atai and D. J. Steigmann, Coupled deformations of elastic curves and surfaces, Int. J. Solids Structures 35(16), 1915-1952 (1998)

[Bag96] F. Baginski, Modeling nonaxisymmetric off-design shapes of large scientific balloons, AIAA Journal 34(2), 400-407 (1996)

[BB98] F. Baginski and K. A. Brakke, Modeling ascent configurations of strained high altitude balloons, AIAA Journal 36(10), 1901-1910 (1998)

[BC98] F. Baginski and W. Collier, A mathematical model for the strained shape of a large scientific balloon at float altitude. Manuscript, George Washington University, 1988, J. Appl. Mech., Vol. 67 , issue 1, pp. 6-16, 2000

[BR95] F. Baginski and S. Ramamurti, Variational principles for ascent shapes of large scientific balloons, AIAA Journal 33(4) 764-768 (1995)

[Cia88] P. Ciarlet, Mathematical Elasticity. Vol. 1, Elsevier Science Publishers, Amsterdam, 1988

[Dac89] B. Dacorogna, Direct Methods in the Calculus of Variations, Springer-Verlag, 1989

[Fis88] D. Fisher, Configuration dependent pressure potentials, Journal of Elasticity 19, 77-84, 1988

[HS94] E. M. Haseganu and D. J. Steigmann, Analysis of partly wrinkled membranes by the method of dynamic relaxation, Computational Mechanics 14, 596-614 (1994)

[Pip86] A. C. Pipkin, The relaxed energy density for isotropic elastic membranes, IMA Journal of Applied Mathematics 36, 85-99 (1986)

[Pip94] A. C. Pipkin, Relaxed energy densities for large deformations of membranes, IMA Journal of Applied Mathematics 52, 297-308 (1994)

[Sch91] W. W. Schur, Structural behavior of scientific balloons; finite element simulation and verification, 1991. AIAA-91-3668-CP

[Sch97] W. W. Schur, Structural response of a zero-pressure balloon with an axial load tendon. In AIAA International Balloon Technology Conference. AIAA, June 1997

[Sou94] A. Soubrier, French contribution to new balloon designs and materials. Adv. Space Res. 14(2), (2)5-(2)12 (1994)

[Ste91a] D. J. Steigmann, A note on pressure potentials, Journal of Elasticity 26, 87-93 (1991)

[Ste91b] D. J. Steigmann, Tension-field theories of elastic membranes and networks, AMD 124, 41-49 (1991) 